\title{
An elementary property of correlations
}

\author{
Giovanni Coppola
}

To the memory of S. Srinivasan

\begin{abstract}
We study the "shift-Ramanujan expansion" to obtain a formulae for the shifted convolution sum $C_{f, g}(N, a)$ of general functions $f, g$ satisfying Ramanujan Conjecture; here, the shift-Ramanujan expansion is with respect to a shift factor $a>0$. Assuming Delange Hypothesis for the correlation, we get the "Ramanujan exact explicit formula", a kind of finite shift-Ramanujan expansion. A noteworthy case is when $f=g=\Lambda$, the von Mangoldt function; so $C_{\Lambda, \Lambda}(N, 2 k)$, for natural $k$, corresponds to $2 k$-twin primes; under the assumption of Delange Hypothesis, we easily obtain the proof of Hardy-Littlewood Conjecture for this case.
\end{abstract}

Keywords. correlation, shift Ramanujan expansion, $2 k$-twin primes

2010 Mathematics Subject Classification. 11N05, 11P32, 11N37

\section{Introduction and statement of the results}

We define for any arithmetic functions $f, g: \mathbb{N} \rightarrow \mathbb{C}$ their correlation (or shifted convolution sum) of shift a:

$$
C_{f, g}(N, a) \stackrel{\text { def }}{=} \sum_{n \leq N} f(n) g(n+a), \text { for all } a \in \mathbb{N} .
$$

Notice in passing that it is an arithmetic function itself, of argument $a \in \mathbb{N}$, the shift. In fact, in $\S 5$ of [CoMu18] we introduced the shift-Ramanujan expansion, i.e. (see (1) in [CoMu18] for $c_{\ell}(a)$, the Ramanujan sum):

$$
C_{f, g}(N, a)=\sum_{\ell=1}^{\infty} \widehat{C_{f, g}}(N, \ell) c_{\ell}(a), \text { for all } a \in \mathbb{N} .
$$

Any arithmetic function $F: \mathbb{N} \rightarrow \mathbb{C}$ may be written as $F(n)=\sum_{d \mid n} F^{\prime}(d)$, by Möbius inversion [Ten95], with a uniquely determined $F^{\prime} \stackrel{\text { def }}{=} F * \mu$ (see [Ten95] for $*, \mu$ ), its Eratosthenes transform (Wintner's [Win43] terminology).

We shall, hereafter, truncate $g(m)=\sum_{q \mid m} g^{\prime}(q)$ as $g_{N}(m) \stackrel{\text { def }}{=} \sum_{q \mid m, q \leq N} g^{\prime}(q)$; in fact, our calculations will be shorter, with an $a$-independent truncation at a small cost, i.e. the error is small:

$$
C_{f, g}(N, a)-C_{f, g_{N}}(N, a)=\sum_{N<q \leq N+a} g^{\prime}(q) \sum_{\substack{n \leq N \\ n \equiv-a \bmod q}} f(n) \ll \max _{n \leq N}|f(n)| \cdot \max _{N<q \leq N+a}\left|g^{\prime}(q)\right| \cdot a, \text { for all } a \in \mathbb{N},
$$

which, in the case $f$ and $g$ satisfy the Ramanujan Conjecture, ${ }^{1}$ is $O_{\varepsilon}\left(N^{\varepsilon}(N+a)^{\varepsilon} a\right)$, uniformly for all $a \in \mathbb{N}$.

\footnotetext{
We thank episciences.org for providing open access hosting of the electronic journal Hardy-Ramanujan Journal

${ }^{1}$ Ramanujan Conjecture for $f$ says: $f(n) \ll_{\varepsilon} n^{\varepsilon}$, as $n \rightarrow \infty$. Hereafter Vinogradov's $\ll$ is equivalent to Landau's $O$-notation, [Ten95], also, $\ll_{\varepsilon}$ says, like $O_{\varepsilon}$, that the constant may depend on arbitrarily small $\varepsilon>0$.
} 
We say, by definition, that a correlation $C_{f, g}(N, a)$ is fair when the dependence on the shift $a$ is only inside the argument of $g, n+a$, but not in $f, g$, neither in their supports. Assuming " $g$ has range $Q$ ", i.e.,

$$
g(m)=g_{Q}(m) \stackrel{\text { def }}{=} \sum_{q \mid m, q \leq Q} g^{\prime}(q)=\sum_{\ell \leq Q} \hat{g}(\ell) c_{\ell}(m), \text { where } \hat{g}(\ell) \stackrel{\text { def }}{=} \sum_{q \equiv 0 \bmod \ell} \frac{g^{\prime}(q)}{q}
$$

(that is, compare [CoMuSa17], $g_{Q}$ finite Ramanujan expansion), with $Q$ independent of $a$, then $C_{f, g}(N, a)$ is

$$
C_{f, g}(N, a)=C_{f, g_{Q}}(N, a)=\sum_{q \leq Q} \hat{g}(q) \sum_{n \leq N} f(n) c_{q}(n+a), \text { for all } a \in \mathbb{N}
$$

where the $\hat{g}(q)$ are above Ramanujan coefficients of $g$. This correlation is fair if and only if all the $f(n)$, the $\hat{g}(q)$ and their supports don't depend on $a$, i.e.: $a$-dependence is only in $c_{q}(n+a)$ ! We define:

$$
C_{f, g_{N}}^{\prime}(N, \ell) \stackrel{\text { def }}{=} \sum_{t \mid \ell} C_{f, g_{N}}(N, t) \mu\left(\frac{\ell}{t}\right)
$$

which has part in the following Delange Hypothesis, for the truncated correlation $C_{f, g_{N}}(N, a)$ :

$$
\sum_{d=1}^{\infty} \frac{2^{\omega(d)}}{d}\left|C_{f, g_{N}}^{\prime}(N, d)\right|<\infty
$$

where the arithmetic function $\omega(d)$ counts the prime factors of $d$, whence $2^{\omega(d)}$ is the number of square-free divisors of $d$, that has bound

$$
2^{\omega(d)} \ll_{\varepsilon} d^{\varepsilon}, \text { as } d \rightarrow \infty,
$$

since it is bounded by the number of divisors of $d$ (and divisor function also satisfies Ramanujan Conjecture).

It is very well known that for general arithmetic functions (see following result) Delange Hypothesis implies Carmichael's Formula: here we apply this to our truncated correlation, getting for it, from the above hypothesis (DH), the formula

$$
\widehat{C_{f, g_{N}}}(N, \ell)=\frac{1}{\varphi(\ell)} \lim _{x \rightarrow \infty} \frac{1}{x} \sum_{a \leq x} C_{f, g_{N}}(N, a) c_{\ell}(a)
$$

where $\varphi(\ell) \stackrel{\text { def }}{=}|\{n \leq \ell:(n, \ell)=1\}|$ is the Euler function. Actually, the implication $(\mathrm{DH}) \Rightarrow(\mathrm{CF})$ follows from a result of Wintner (of 1943 [Win43]) and a result of Delange (published in 1976, [De76]) that we quote here from [ScSp94] Theorem 2.1 in Chapter VIII on Ramanujan expansions (restating and selecting properties), for all arithmetic functions $F$ :

Wintner-Delange Formula. Let $F: \mathbb{N} \rightarrow \mathbb{C}$ satisfy Delange Hypothesis, namely

$$
\sum_{d=1}^{\infty} \frac{2^{\omega(d)}}{d}\left|F^{\prime}(d)\right|<\infty
$$

Then the Ramanujan expansion

$$
\sum_{q=1}^{\infty} \widehat{F}(q) c_{q}(n)
$$


converges pointwise to $F(n)$, for all $n \in \mathbb{N}$, with coefficients given by the formula

$$
\widehat{F}(q)=\sum_{d \equiv 0 \bmod q} \frac{F^{\prime}(d)}{d}, \text { for all } q \in \mathbb{N}
$$

(where the series on $R H S$, right hand side, converges pointwise, for all $q \in \mathbb{N}$ ) and also by Carmichael ${ }^{2}$ formula

$$
\widehat{F}(q)=\frac{1}{\varphi(q)} \lim _{x \rightarrow \infty} \frac{1}{x} \sum_{n \leq x} F(n) c_{q}(n), \text { for all } q \in \mathbb{N}
$$

(where the limit on $R H S$ exists, for all $q \in \mathbb{N}$ )

We don't need, actually, to prove this result, as it follows from (quoted) Th.2.1 of [ScSp94]. In the case $F(a)=C_{f, g_{N}}(N, a)$, assuming the above (DH) (i.e., Delange Hypothesis for present $F$ ), then Wintner-Delange formula implies the above $(\mathrm{CF})$ (i.e., Carmichael Formula for $F$ ); this, in turn, is condition (ii) of Theorem 1 in [CoMu18] which is equivalent, choosing $Q=N$, to the following $\mathbf{R}$ amanujan exact explicit formula (as we named condition (iii) in Theorem 1 [CoMu18]) for $C_{f, g_{N}}$, that is also uniform in $a \in \mathbb{N}$ :

$$
C_{f, g_{N}}(N, a)=\sum_{\ell \leq N}\left(\frac{\hat{g}(\ell)}{\varphi(\ell)} \sum_{n \leq N} f(n) c_{\ell}(n)\right) c_{\ell}(a) .
$$

R.e.e.f.

This part of our original correlation $C_{f, g}$, for general $f, g: \mathbb{N} \rightarrow \mathbb{C}$ satisfying Ramanujan Conjecture, has a lot of structure (it's a truncated divisor sum!); adding the other part, we estimated above in (1), we get, for fair correlations with $(\mathrm{DH})$, the following "structure + small error" - elementary property (that gives name to the paper).

Theorem. Let $f, g: \mathbb{N} \rightarrow \mathbb{C}$ satisfy the Ramanujan Conjecture and be such that, for the $N$-truncated divisor sum $g_{N}(m)$ defined above, the correlation $C_{f, g_{N}}$ is fair and satisfies (DH). Then

$$
C_{f, g}(N, a)=\sum_{\ell \leq N}\left(\frac{\hat{g}(\ell)}{\varphi(\ell)} \sum_{n \leq N} f(n) c_{\ell}(n)\right) c_{\ell}(a)+O_{\varepsilon}\left(N^{\varepsilon}(N+a)^{\varepsilon} a\right),
$$

uniformly in $a \in \mathbb{N}$.

What we said up to now suffices to prove the Theorem (notice: (1) \& (2), Wintner-Delange result above and Theorem 1 in [CoMu18] are the whole proof ). QED $^{3}$

However, thanks to the importance and generality (in $\S 3$ we have, say, a huge application too) we will provide a step-by-step proof in next section, $\S 2$.

In a perfectly similar fashion to the proof of Corollary 1 [CoMu18], from Theorem 1 [CoMu18], we can prove (but we will not do) the following consequence.

Corollary 1. Assume $f, g: \mathbb{N} \rightarrow \mathbb{C}$ satisfy Ramanujan Conjecture, where furthermore $f$ is a $D$ truncated divisor sum, say $f(n)=f_{D}(n) \stackrel{\text { def }}{=} \sum_{d \mid n, d \leq D} f^{\prime}(d)$, with $\frac{\log D}{\log N}<1-\delta$. Also, let the correlation $C_{f, g_{N}}$ be fair, with (DH). Then

$$
C_{f, g}(N, a)=\mathfrak{S}_{f, g}(a) N+O\left(N^{1-\delta}\right)+O_{\varepsilon}\left(N^{\varepsilon}(N+a)^{\varepsilon} a\right)
$$

\footnotetext{
${ }^{2}$ The name given here is in honour of Carmichael [Ca32]: compare [Mu13, pp.26-27] for details

${ }^{3}$ In this paper, QED(=Quod Erat Demonstrandum=What was to be shown) is not the end of the story, in a proof (we use $\square$ for it); also, in the following, it will indicate an involved, smaller, part of proof ending
} 
uniformly in $a \in \mathbb{N}$, where the "singular sum", here, is defined with $f, g$ Ramanujan coefficients as

$$
\mathfrak{S}_{f, g}(a) \stackrel{\text { def }}{=} \sum_{q \leq N} \hat{f}(q) \hat{g}(q) c_{q}(a), \text { for all } a \in \mathbb{N} .
$$

Before an "unnecessary", but beautiful, proof of our Theorem (that, actually, will prove even the above Wintner-Delange formula), we apply our Theorem, in section $\S 3$, to the noteworthy case of $2 k$-twin primes, assuming (DH) for them. Also, we realised later that this noteworthy case also comes from our Theorem 1 [CoMu18]. In fact, truncating $g$ at $Q=N$ (in Theorem 1) and considering a kind of approximation, to original correlation, as given above in equation (1), everything works fine!

\section{The detailed proof of our Theorem}

Proof. Starting from (1), we are left with the task of proving the R.e.e.f. above, i.e.,

$$
\sum_{n \leq N} f(n) \sum_{q \mid n+a, q \leq N} g^{\prime}(q)=\sum_{\ell \leq N}\left(\frac{\hat{g}(\ell)}{\varphi(\ell)} \sum_{n \leq N} f(n) c_{\ell}(n)\right) c_{\ell}(a) .
$$

The hypotheses of our Theorem ensure that the LHS, namely $C_{f, g_{N}}(N, a)$, satisfies (DH) above. Now, we need to infer $(\mathrm{DH}) \Rightarrow(\mathrm{CF})$ (see the above), namely, get the Carmichael formula for our $C_{f, g_{N}}(N, a)$, so to have in the following, say, a way to infer the R.e.e.f.! However, we'll supply even more, by providing a proof, for the above "Wintner-Delange formula". (Hence, in the immediate following we'll import arguments from [De76] \& [ScSp94].)

In order to prove it, we wish to prove that the following double series, over $\ell, d$ summations, is absolutely convergent; so, we may write the equation expressing it in two ways (first summing over $\ell$, then $d$ and the vice versa):

$$
\sum_{d=1}^{\infty} \sum_{\ell \mid d} \frac{F^{\prime}(d)}{d} c_{\ell}(n)=\sum_{\ell=1}^{\infty} \sum_{d \equiv 0 \bmod \ell} \frac{F^{\prime}(d)}{d} c_{\ell}(n), \text { for all } n \in \mathbb{N},
$$

namely, exchange sums. In fact, $\frac{1}{d} \sum_{\ell \mid d} c_{\ell}(n)=\mathbf{1}_{d \mid n}$, for $\mathbf{1}_{\wp}=1$ if and only if $\wp$ is true (0 otherwise), [CoMuSa17, Lemma 1] gives LHS

$$
\sum_{d=1}^{\infty} \frac{F^{\prime}(d)}{d} \sum_{\ell \mid d} c_{\ell}(n)=\sum_{d \mid n} F^{\prime}(d)=F(n),
$$

with on RHS the Wintner-Delange coefficients

$$
\sum_{d \equiv 0 \bmod \ell} \frac{F^{\prime}(d)}{d}, \text { for all } \ell \in \mathbb{N}
$$

thus supplying a proof of the first (Wintner-Delange's!) formula and also ensuring pointwise convergence of Ramanujan expansion, with these coefficients:

$$
(*) \Rightarrow F(n)=\sum_{\ell=1}^{\infty}\left(\sum_{d \equiv 0 \bmod \ell} \frac{F^{\prime}(d)}{d}\right) c_{\ell}(n), \text { for all } n \in \mathbb{N} .
$$


Absolute convergence of double series comes from the fact that LHS with moduli, for all $d, \ell \in \mathbb{N}$, are bounded by

$$
\sum_{d=1}^{\infty} \frac{\left|F^{\prime}(d)\right|}{d} \sum_{\ell \mid d}\left|c_{\ell}(n)\right| \leq n \sum_{d=1}^{\infty} \frac{\left|F^{\prime}(d)\right|}{d} 2^{\omega(d)}<\infty, \text { for all } n \in \mathbb{N},
$$

coming as we know from Delange Hypothesis, starting from the optimal bound, proved by Hubert Delange:

$$
\sum_{\ell \mid d}\left|c_{\ell}(n)\right| \leq n \cdot 2^{\omega(d)}
$$

for which we refer to Delange's original paper [De76] (also, for comments about optimality).

Left to prove, for Wintner-Delange formula above, is the fact that above coefficients (WintnerDelange's, which we know, now, to be our Ramanujan coefficients!) are given also by the Carmichael formula:

$$
\frac{1}{\varphi(q)} \lim _{x \rightarrow \infty} \frac{1}{x} \sum_{n \leq x} F(n) c_{q}(n)=\sum_{d \equiv 0 \bmod q} \frac{F^{\prime}(d)}{d},
$$

our task, now; for which we plug (in LHS), for a large $K \in \mathbb{N}$, the decomposition:

$$
F(n)=\sum_{d \mid n, d \leq K} F^{\prime}(d)+\sum_{d \mid n, d>K} F^{\prime}(d)
$$

rendering in the LHS the following (again, sums exchange is possible because $F^{\prime}$ can't depend on $n$ ):

$$
\frac{1}{x} \sum_{n \leq x} F(n) c_{q}(n)=\sum_{d \leq K} F^{\prime}(d) \frac{1}{x} \sum_{m \leq x / d} c_{q}(d m)+\sum_{d>K} F^{\prime}(d) \frac{1}{x} \sum_{m \leq x / d} c_{q}(d m),
$$

in which, now, we apply two different treatments, depending on $d \leq K$ or $d>K$. For low divisors $d$,

$$
\begin{gathered}
\sum_{d \leq K} F^{\prime}(d) \frac{1}{x} \sum_{m \leq x / d} c_{q}(d m)=\sum_{d \leq K} F^{\prime}(d) \sum_{j \leq q,(j, q)=1} \frac{1}{x} \sum_{m \leq x / d} e_{q}(j d m) \\
=\sum_{d \leq K} F^{\prime}(d) \sum_{j \leq q,(j, q)=1}\left(\frac{1}{d} \cdot \mathbf{1}_{d \equiv 0 \bmod q}+O\left(\frac{1}{x}\left(1+\frac{\mathbf{1}_{d \neq 0 \bmod q}}{\left\|\frac{j d}{q}\right\|}\right)\right)\right)=\varphi(q) \sum_{\substack{d \leq K \\
d \equiv 0 \bmod q}} \frac{F^{\prime}(d)}{d}+O(1 / x),
\end{gathered}
$$

from familiar exponential sums cancellations, with a final $O$-constant not affecting the $x$-decay, while for high divisors $d$ :

$$
\sum_{d>K} F^{\prime}(d) \frac{1}{x} \sum_{m \leq x / d} c_{q}(d m) \ll \varphi(q) \sum_{d>K} \frac{\left|F^{\prime}(d)\right|}{d},
$$

uniformly in $x>0$, using the trivial bound $\left|c_{q}(n)\right| \leq \varphi(q)$, for all $n \in \mathbb{Z}$. In all,

$$
\frac{1}{x} \sum_{n \leq x} F(n) c_{q}(n)=\varphi(q) \sum_{\substack{d \leq K \\ d \equiv 0 \bmod q}} \frac{F^{\prime}(d)}{d}+O(1 / x)+O\left(\varphi(q) \sum_{d>K} \frac{\left|F^{\prime}(d)\right|}{d}\right)
$$

entailing

$$
\frac{1}{\varphi(q)} \lim _{x \rightarrow \infty} \frac{1}{x} \sum_{n \leq x} F(n) c_{q}(n)=\sum_{\substack{d \leq K \\ d \equiv 0 \bmod q}} \frac{F^{\prime}(d)}{d}+O\left(\sum_{d>K} \frac{\left|F^{\prime}(d)\right|}{d}\right),
$$

actually, giving the required equation, since from Delange Hypothesis the series $\sum_{d=1}^{\infty} \frac{\left|F^{\prime}(d)\right|}{d}$ converges, so errors in $O$ are infinitesimal with $K$, an arbitrarily large natural number (also, present LHS doesn't 
depend on it!). At last, this also proves the convergence in RHS of these, say, $d \leq K$-coefficients (as $K \rightarrow \infty)$.

QED (Wintner-Delange Formula)

Let's turn to the application of this formula to our case $F(a)=C_{f, g_{N}}(N, a)$, getting that (since we are assuming (DH) in hypotheses) we have the Carmichael formula, (CF) above. Now (mimicking the proof of [CoMu18] Theorem 1, $(i i) \Rightarrow($ iii $)$, exactly) we'll get the Reef above; in fact, let's calculate, since we know that the shift Ramanujan expansion converges (again, from (DH) implying this by just proved Wintner-Delange), its shift-Ramanujan coefficients, for correlation $C_{f, g_{N}}(N, a)$, namely

$$
\widehat{C_{f, g_{N}}}(N, \ell)=\frac{1}{\varphi(\ell)} \lim _{x \rightarrow \infty} \frac{1}{x} \sum_{a \leq x} C_{f, g_{N}}(N, a) c_{\ell}(a) .
$$

Plugging (2) with $Q=N$ inside this RHS, we get for it:

$$
\frac{1}{x} \sum_{a \leq x} C_{f, g_{N}}(N, a) c_{\ell}(a)=\sum_{q \leq N} \hat{g}(q) \sum_{n \leq N} f(n) \frac{1}{x} \sum_{a \leq x} c_{q}(n+a) c_{\ell}(a)
$$

present exchange of sums being possible thanks to the hypothesis: $C_{f, g_{N}}(N, a)$ is fair. Then,

$$
\frac{1}{\varphi(\ell)} \lim _{x \rightarrow \infty} \frac{1}{x} \sum_{a \leq x} C_{f, g_{N}}(N, a) c_{\ell}(a)=\frac{1}{\varphi(\ell)} \sum_{q \leq N} \hat{g}(q) \sum_{n \leq N} f(n) \lim _{x \rightarrow \infty} \frac{1}{x} \sum_{a \leq x} c_{q}(n+a) c_{\ell}(a),
$$

since all we are exchanging with $\lim _{x \rightarrow \infty}$ are finite sums (again, we're implicitly using fairness); then, the orthogonality of Ramanujan sums (first proved by Carmichael in [Ca32], that's why (CF) bears his name), namely Theorem 1 in [Mu13]:

$$
\lim _{x \rightarrow \infty} \frac{1}{x} \sum_{a \leq x} c_{q}(n+a) c_{\ell}(a)=\mathbf{1}_{q=\ell} \cdot c_{q}(n), \text { for all } \ell, n, q \in \mathbb{N},
$$

gives inside $(* *)$ whence for quoted $(\mathrm{CF})$ the shift-Ramanujan coefficients

$$
\widehat{C_{f, g_{N}}}(N, \ell)=\frac{1}{\varphi(\ell)} \hat{g}(\ell) \sum_{n \leq N} f(n) c_{\ell}(n)
$$

and this, thanks to the finite support of $\hat{g}$, up to $Q=N$, here, gives the R.e.e.f.! QED

One last detail: equation (2), actually, we didn't prove; but it follows from $m=n+a$ in (another unproven)

$$
\sum_{q \mid m, q \leq Q} g^{\prime}(q)=\sum_{\ell \leq Q} \hat{g}(\ell) c_{\ell}(m)
$$

that is: the $g_{Q}$ (see the beginning of the paper) finite Ramanujan expansion (f.R.e.) (for which we referred to [CoMuSa17], of course), with Ramanujan coefficients

$$
\hat{g}(\ell) \stackrel{\text { def }}{=} \sum_{q \equiv 0 \bmod \ell} \frac{g^{\prime}(q)}{q} .
$$

This can be proved at once, from quoted Lemma 1 of [CoMuSa17], that we also prove (briefly) here:

$$
\mathbf{1}_{q \mid m}=\frac{1}{q} \sum_{\ell \mid q} c_{\ell}(m),
$$


because: the orthogonality of additive characters [Da00] (rearranging by g.c.d.) gives

$\mathbf{1}_{q \mid m}=\frac{1}{q} \sum_{r \leq q} e_{q}(r m)=\frac{1}{q} \sum_{\ell \mid q} \sum_{r \leq q,(r, q)=q / \ell} e_{q}(r m)=\frac{1}{q} \sum_{\ell \mid q} \sum_{j \leq \ell,(j, \ell)=1} e_{\ell}(j m)$, with $c_{\ell}(n) \stackrel{\text { def }}{=} \sum_{\substack{j \leq \ell \\(j, \ell)=1}} e_{\ell}(j n)$.

Then from this divisibility condition we prove $g_{Q}$ f.R.e.:

$$
\sum_{q \mid m, q \leq Q} g^{\prime}(q)=\sum_{q \leq Q} \frac{g^{\prime}(q)}{q} \sum_{\ell \mid q} c_{\ell}(m)=\sum_{\ell \leq Q} \hat{g}(\ell) c_{\ell}(m)
$$

simply exchanging sums and using above definition of f.R.e. coefficients, $\hat{g}(q)$. QED (for equation (2), too.)

\section{The well-known case $f=g=\Lambda, a=2 k>0$ of our Theorem: $2 k$-prime-twins}

Assuming (DH) for $f=g=\Lambda$, Hardy-Littlewood heuristic (Conjecture B and (5.26) [HaLi23]) is a Theorem.

Corollary 2. Assuming Delange Hypothesis for $C_{\Lambda, \Lambda_{N}}(N, a)$, i.e.

$$
\sum_{d=1}^{\infty} \frac{2^{\omega(d)}}{d}\left|C_{\Lambda, \Lambda_{N}}^{\prime}(N, d)\right|<\infty
$$

we get a kind of Hardy-Littlewood asymptotic formula, with an absolute constant $c>0$, for all fixed $k \in \mathbb{N}$

$$
C_{\Lambda, \Lambda}(N, 2 k)=\mathfrak{S}_{\Lambda, \Lambda}(2 k) N+O\left(N e^{-c \sqrt{\log N}}\right) .
$$

Proof. We apply the calculations for Ramanujan coefficients of the $N$-truncated von Mangoldt function $\Lambda_{N}$, from the classical [Da00] von Mangoldt $\Lambda=(-\mu \log ) * \mathbf{1}$, [Ten95], defined as usual in terms of primes $p \in \mathbb{P}$ :

$$
\Lambda(n) \stackrel{\text { def }}{=} \sum_{k \in \mathbb{N}} \sum_{p \in \mathbb{P}} \mathbf{1}_{n=p^{k}} \log p \Rightarrow \Lambda(n)=\sum_{d \mid n}(-\mu(d) \log d), \Lambda_{N}(n)=\sum_{d \mid n, d \leq N}(-\mu(d) \log d),
$$

entailing

$$
\Lambda_{N}(n)=\sum_{q \leq N} \widehat{\Lambda_{N}}(q) c_{q}(n), \quad \widehat{\Lambda_{N}}(q) \stackrel{d e f}{=}-\sum_{\substack{d \leq N \\ d \equiv 0 \bmod q}} \frac{\mu(d) \log d}{d} \ll \frac{\log ^{2} N}{q},
$$

where now these are, thanks to $\S 4$ of [CoMu18], with an absolute $c>0$,

$$
\widehat{\Lambda_{N}}(q)=\frac{\mu(q)}{\varphi(q)}+O\left(\frac{1}{q} \exp (-c \sqrt{\log N})\right), \text { for all } q \leq \sqrt{N},
$$

thanks to the zero-free region of Riemann zeta-function (actually, we are not using most recent one). Now,

$$
C_{\Lambda, \Lambda}(N, a)=\sum_{\ell \leq N} \frac{\widehat{\Lambda_{N}}(\ell)}{\varphi(\ell)}\left(\sum_{n \leq N} \Lambda(n) c_{\ell}(n)\right) c_{\ell}(a)+O_{\varepsilon}\left(N^{\varepsilon}(N+a)^{\varepsilon} a\right),
$$


from our Theorem: $C_{\Lambda, \Lambda_{N}}$ is fair \& assume $(\mathrm{DH}), f=g=\Lambda$. Set $a=2 k>0, \frac{\log k}{\log N}<1-\delta$, $\delta \in(0,1 / 2)$ fixed:

$$
\begin{gathered}
C_{\Lambda, \Lambda}(N, a)=\sum_{\ell \leq \sqrt{N}} \frac{\mu(\ell)}{\varphi^{2}(\ell)}\left(\sum_{n \leq N} \Lambda(n) c_{\ell}(n)\right) c_{\ell}(a)+O\left(\exp (-c \sqrt{L}) \sum_{\ell \leq \sqrt{N}} \frac{(a, \ell)}{\ell \varphi(\ell)} \sum_{n \leq N} \Lambda(n)(n, \ell)\right) \\
+O\left(L^{2} \sum_{\sqrt{N<\ell \leq N}} \frac{(a, \ell)}{\ell \varphi(\ell)} \sum_{n \leq N} \Lambda(n)(n, \ell)\right)+O\left(N^{1-\delta}\right),
\end{gathered}
$$

where we have applied well-known $\left|c_{q}(n)\right| \leq(q, n)$, see Lemma A.1 in [CoMu18], and above bounds for $\Lambda_{N}$, abbreviating hereafter $L \stackrel{\text { def }}{=} \log N$. In the main term, applying PNT(Prime Number Theorem) [Da00], [Ten95]:

$$
\sum_{n \leq N} \Lambda(n) c_{\ell}(n)=\mu(\ell) \sum_{\substack{n \leq N \\(n, \ell)=1}} \Lambda(n)+O\left(L \varphi(\ell) \sum_{p \mid \ell} \log p\right) \stackrel{\mathrm{PNT}}{=} \mu(\ell) N+O\left(N e^{-c \sqrt{L}}\right)+O(L \varphi(\ell) \log \ell),
$$

from well known [Da00]: $\quad \sum_{p \mid \ell} \log p \leq \sum_{n \mid \ell} \Lambda(n)=\log \ell$; here, we need to bound the $n$-sum in remainders as

$\sum_{n \leq N} \Lambda(n)(n, \ell)=\sum_{d \mid \ell} d \sum_{\substack{n \leq N \\(n, \ell)=d}} \Lambda(n) \ll \sum_{d \mid \ell} d \sum_{\substack{n \leq N \\ n \equiv 0 \bmod d}} \Lambda(n) \ll N+\ell L \sum_{k \in \mathbb{N}} \sum_{p^{k} \mid \ell} \log p \ll N L^{2}$, for all $\ell \leq N$,

by Čebičev bound [Ten95]: $\quad \sum_{n \leq N} \Lambda(n) \ll N$. Then, using [Ten95]: $\quad \varphi(\ell) \gg \ell / \log \ell$, changing time to time $c>0$,

$$
\begin{aligned}
& C_{\Lambda, \Lambda}(N, a)=N \sum_{\ell \leq \sqrt{N}} \frac{\mu^{2}(\ell)}{\varphi^{2}(\ell)} c_{\ell}(a)+O\left(N e^{-c \sqrt{L}} \sum_{\ell \leq \sqrt{N}} \frac{(a, \ell)}{\ell^{2}}+N L^{5} \sum_{\sqrt{N}<\ell \leq N} \frac{(a, \ell)}{\ell^{2}}+N^{1-\delta}\right) \\
& \quad=N \sum_{\ell=1}^{\infty} \frac{\mu^{2}(\ell)}{\varphi^{2}(\ell)} c_{\ell}(a)+O\left(N \sum_{\ell>\sqrt{N}} \frac{\log ^{2} \ell}{\ell^{2}}(a, \ell)\right)+O\left(N e^{-c \sqrt{L}} \sum_{\ell \leq \sqrt{N}} \frac{(a, \ell)}{\ell^{2}}+N L^{5} \sum_{\sqrt{N}<\ell \leq N} \frac{(a, \ell)}{\ell^{2}}+N^{1-\delta}\right)
\end{aligned}
$$

being, by the definition of classic singular series for $a=2 k$-twin primes,

$$
\mathfrak{S}_{\Lambda, \Lambda}(a) \stackrel{\text { def }}{=} \sum_{\ell=1}^{\infty} \frac{\mu^{2}(\ell)}{\varphi^{2}(\ell)} c_{\ell}(a)
$$

and, also, by following bounds: (use $(A+B)^{2} \ll A^{2}+B^{2}$, then, [Ten95]: $\sum_{d \mid a} 1 \ll_{\varepsilon} a^{\varepsilon}$ and $\left.\sum_{d \leq x} 1 / d \ll \log x\right)$

$$
\begin{aligned}
& \sum_{\ell>\sqrt{N}} \frac{\log ^{2} \ell}{\ell^{2}}(a, \ell) \ll \sum_{\substack{d \mid a \\
d \leq \sqrt{N}}} \frac{1}{d} \sum_{m>\sqrt{N} / d} \frac{\log ^{2} d+\log ^{2} m}{m^{2}}+\sum_{\substack{d \mid a \\
d>\sqrt{N}}} \frac{1}{d} \sum_{m=1}^{\infty} \frac{\log ^{2} d+\log ^{2} m}{m^{2}} \ll_{\varepsilon} a^{\varepsilon} \frac{L^{2}}{\sqrt{N}}, \\
& \sum_{\ell \leq \sqrt{N}} \frac{(a, \ell)}{\ell^{2}} \ll \sum_{\substack{d \mid a \\
d \leq \sqrt{N}}} \frac{1}{d} \sum_{m \leq \sqrt{N} / d} \frac{1}{m^{2}} \ll L \\
& \sum_{\sqrt{N}<\ell \leq N} \frac{(a, \ell)}{\ell^{2}} \ll \sum_{\substack{d \mid a \\
d \leq \sqrt{N}}} \frac{1}{d} \sum_{\sqrt{N} / d<m \leq N / d} \frac{1}{m^{2}}+\sum_{\substack{d \mid a \\
d>\sqrt{N}}} \frac{1}{d} \sum_{m \leq N / d} \frac{1}{m^{2}} \ll \varepsilon \frac{a^{\varepsilon}}{\sqrt{N}}
\end{aligned}
$$


uniformly in $a=2 k, k \in \mathbb{N}$, with $\frac{\log k}{\log N}<1-\delta$, for a fixed $\delta \in(0,1 / 2)$, proves Hardy-Littlewood Conjecture $^{4}$

$$
C_{\Lambda, \Lambda}(N, 2 k)=\mathfrak{S}_{\Lambda, \Lambda}(2 k) N+O\left(N e^{-c \sqrt{\log N}}\right)
$$

We don't have time to go deeper (but we've plenty of margins ${ }^{5}$ ).

Acknowledegments. I wish to thank Ram Murty, not only for the biggest part of the work laying behind present Theorem \& Corollary 1 (coming from [CoMu18] of course), but also for the real beginning of my interest in Ramanujan expansions and their applications to analytic number theory, thanks to his "illuminating" survey [Mu13] on "Ramanujan series", ironically (in the good meaning), leading then to finite Ramanujan expansions!

Also I wish to thank the Referee for having improved the paper, thanks to precious hints.

\section{References}

[Ca32] Carmichael, R.D., Expansions of arithmetical functions in infinite series, Proc. London Math. Society 34 (1932), 1-26. MR 1576142

[CoMuSa17] Coppola, G. and Murty, M.Ram and Saha, B., Finite Ramanujan expansions and shifted convolution sums of arithmetical functions J. Number Theory 174 (2017), 78-92.

[CoMu18] Coppola, G. and Murty, M.Ram, Finite Ramanujan expansions and shifted convolution sums of arithmetical functions, II, J. Number Theory 185 (2018), 16-47.

[Da00] Davenport, H., Multiplicative Number Theory, Third Edition, GTM 74, Springer, New York, 2000. MR 2001f:11001

[De76] Delange, H., On Ramanujan expansions of certain arithmetical functions, Acta Arith. 31 (1976), 259-270. MR 432578

[HaLi23] Hardy, G.H. and Littlewood, J.E., Some problems of "Partitio numerorum". III: On the expression of a number as a sum of primes, Acta Math. 44 (1923), 1-70.

[Mu13] Murty, M.Ram, Ramanujan series for arithmetical functions, Hardy-Ramanujan J., 36 (2013), 21-33.

[ScSp94] Schwarz, W. and Spilker, J., Arithmetical functions, (An introduction to elementary and analytic properties of arithmetic functions and to some of their almost-periodic properties), London Mathematical Society Lecture Note Series, 184, Cambridge University Press, Cambridge, 1994. MR 1274248

[Ten95] Tenenbaum, G., Introduction to Analytic and Probabilistic Number Theory, Cambridge Studies in Advanced Mathematics, 46, Cambridge University Press, 1995. MR 97e:11005b

[Win43] Wintner, A., Eratosthenian averages, Waverly Press, Baltimore, MD, 1943. MR0015082

\section{Giovanni Coppola}

Università degli Studi di Salerno

Home address: Via Partenio 12

83100, Avellino (AV) - ITALY

e-mail: giovanni.coppola@unina.it

\footnotetext{
${ }^{4}$ In this version, it's actually a strong form of famous Conjecture!

${ }^{5}$ In 1637 Fermat wrote “... Hanc marginis exiguitas non caperet."
} 